\title{
Opinião de consumidores do município de Campinas (SP) sobre riscos à saúde provenientes dos alimentos.
}

\author{
Maria da Conceição Pereira da Fonseca ${ }^{1}$, Elisabete Salay ${ }^{2}$
}

\begin{abstract}
Estudos mostram que é expressiva nos países desenvolvidos a preocupação dos consumidores com os riscos alimentares à saúde. Ressaltando-se que esse tipo de preocupação, juntamente com variáveis socioeconômicas e demográficas, pode influenciar no padrão de consumo dos alimentos. No Brasil, os estudos relativos à opinião dos consumidores referente à segurança dos alimentos são ainda restritos. Este estudo avaliou a opinião de consumidores da cidade de Campinas (SP) com relação aos atributos dos alimentos, considerados prejudiciais à saúde. Esta pesquisa é exploratória e compreendeu entrevistas com 158 indivíduos, tendo-se adotado questionários com uma questão dissertativa sobre os riscos alimentares, além de questões que visavam à caracterização socioeconômica e demográfica dos entrevistados. Para a análise estatística, utilizou-se o teste de qui-quadrado $\left(\chi^{2}\right)$ e o cálculo dos resíduos padronizados ( $Z$ res.). Os entrevistados citaram cerca de 20 atributos dos alimentos, considerados prejudiciais à saúde, sendo que a gordura $(24,3 \%)$ e o colesterol $(11,3 \%)$ prevaleceram. Contudo, uma parcela expressiva da população não fez referência aos microorganismos patogênicos. Sugere-se que seja implementada uma política de educação e informação sobre a segurança dos alimentos visando eliminar distorções relativas à percepção dos consumidores.
\end{abstract}

Palavras-chave: segurança do alimento, opinião de consumidor, educação do consumidor.

\section{Consumer opinion about food safety risks in the city of Campinas, SP.}

Studies have shown the consumers' increasing concern in developed countries about health risks associated with food. This fact is remarkable since it can influence the food consumption pattern, along with other socioeconomic and demographic variables. Consumer perception regarding food safety is an area of research relatively unknown in Brazil. This study had the purpose of evaluating the opinion of consumers in the city of Campinas, Brazil, regarding food risks to human health. An exploratory survey was conducted with 158 consumers, by the application of a questionnaire posing a number of open questions regarding risks, and also including the interviewees' characterization. The chi-square test $\left(\chi^{2}\right)$ and the standardized residues calculation ( $Z$ res.) were applied in the statistical analysis. Twenty food attributes were mentioned as harmful to health, among which fat $(24.3 \%)$ and cholesterol $(11.3 \%)$ were the most mentioned. Pathogenic microorganisms did not concern the majority of the population. The results suggest the need to implement education and communication policies to disseminate food safety information to the Brazilian population, in order to correct distortions in the consumers' perception in terms of food safety issues.

Keywords: food safety, consumer opinion, consumer education.

\footnotetext{
${ }^{1}$ Universidade Estadual de Campinas - UNICAMP - Pesquisadora do Núcleo de Estudos e Pesquisas em Alimentação - NEPA, Avenida Albert Einstein, 291 - Cidade Universitária,- Campinas SP, CEP. 13083-852, endereço eletrônico: cfonseca@unicamp.br

${ }^{2}$ Professora Associada da Faculdade de Engenharia de Alimentos - Departamento de Alimentos e Nutrição.

Rua Monteiro Lobato, nº 80 - Cidade Universitária - CAMPINAS - SP CEP. 13083-862 endereço eletrônico: salay@fea.unicamp.br
} 


\section{Introdução}

$\mathrm{Na}$ escolha dos alimentos, os consumidores consideram cada vez mais os riscos que os produtos alimentares podem oferecer à saúde, como os microbiológicos, os causados por práticas higiênicas e métodos de produção impróprios, pela aplicação de pesticidas e também pelo uso da biotecnologia e várias outras inovações tecnológicas (COSTA et al., 2000; SABA et al., 2000; FREWER, 2001; CHINNICI, 2002; O'DONOVAN \& McCARTHY, 2002).

$\mathrm{Na}$ verdade, a relação entre os riscos de origem alimentar e o consumidor é muito complexa e diversos estudos abordam este tema. Dentre as revisões bibliográficas sobre o assunto destacam-se os artigos de Wandel (1994) e Wilcock et al. (2004). A visão econômica que analisa um mercado fictício envolvendo o atributo da segurança do alimento foi desenvolvida por Caswell (1995). Adotando a abordagem psicológica, Yeung e Morris (2001) e Nelson (2004) analisam a relação entre o comportamento de compra dos indivíduos e a percepção do risco. Com enfoque na política pública, Rimal et al. (2001) e Clayton e Griffith (2003), entre outros, analisaram a percepção, a atitude, o conhecimento e o comportamento de indivíduos em relação aos riscos alimentares, discutindo seus possíveis reflexos sobre a saúde da população.

No Brasil, ainda são escassos os trabalhos que envolvem análises relativas ao consumidor e à segurança do alimento ${ }^{3}$. No entanto, segundo Fazio et al. (1997) e Spers (1998) o interesse por pesquisas relativas a esse tema tem crescido. Adotando o método Conjoint Analysis, Spers (1998) avaliou a demanda do consumidor de feira tradicional e de feira orgânica por atributos de segurança do morango. A percepção dos consumidores com relação à qualidade da carne bovina foi estudada por Spers (2003) e Buso (2002). Em uma ampla pesquisa sobre o hábito alimentar no território nacional, foi levantada uma lista de preocupações da população com os alimentos, destacando-se o emprego de conservantes (REVISTA SUPER INTERESSANTE, 1991); em São Paulo, Praxedes (2003) identificou o conhecimento da população sobre higiene e alimentos. Destaca-se ainda que o Instituto Brasileiro de Opinião Pública e EstatísticaIBOPE (2001 e 2002) avaliou a opinião dos consumidores sobre os alimentos transgênicos.

O presente trabalho visa contribuir para a discussão na área de segurança do alimento, no âmbito de programas públicos voltados à informação e educação da população. Para tanto pretende-se identificar a opinião de consumidores sobre atributos dos alimentos relacionados a riscos à saúde e analisá-la, tendo como referência as características socioeconômicas e demográficas de amostra de indivíduos do município de Campinas (SP).

\section{Metodologia}

Campinas é um município paulista, com área de $887 \mathrm{~km}^{2}$ e densidade demográfica de $1.217,83$ habitantes por $\mathrm{km}^{2}$. Sua população é de 1.017 .243 habitantes, com taxa de urbanização 98,5\% (FUNDAÇÃO DO SISTEMA ESTADUAL DE ANÁLISES DE DADOS - SEADE, 2005).

A partir de estudo piloto com 20 entrevistas foi possível estimar uma amostra de tamanho 158 consumidores. Essa amostra foi considerada suficiente para uma análise confiável dos resultados apresentados, uma vez que se obteve um nível de confiança de $95 \%$ e um erro de $0,6 \%$ para as análises gerais da pesquisa. Para as análises socioeconômica e demográfica a confiabilidade obtida foi de $95 \%$, com erro de até $1,3 \%$, tendo-se usado o método do intervalo de confiança para estabelecer que a média esteja contida no intervalo

$$
\bar{x}-t \frac{\sigma}{\sqrt{n}} \leq \mu \leq \bar{x}+t \frac{\sigma}{\sqrt{n}}
$$

onde $n$ é o tamanho da amostra, $\sigma$ é o desvio padrão e $t$ é uma variável aleatória com distribuição $t$-Student, que vale 1,96 para n>120 e uma confiabilidade de $95 \%$.

Os 158 consumidores, maiores de 15 anos, residentes na cidade de Campinas (SP), foram abordados aleatoriamente em locais de grande circulação pública, durante o mês de junho de 2001.

\footnotetext{
${ }^{3}$ Segundo a Associação Brasileira de Normas Técnicas (ABNT) a segurança do alimento é a "garantia de que o produto não contém perigos de natureza biológica, física ou química que possam causar agravos à saúde do consumidor” (ABNT, 2002, p. 3).
} 
Opinião de consumidores e riscos alimentares, Fonseca et al.

O levantamento da opinião dos indivíduos com relação à segurança dos alimentos teve caráter exploratório e foi realizado mediante instrumento de coleta pré-testado que continha, dentre outras, a questão: "Quais as características ou aspectos dos alimentos que você acha que poderiam fazer mal à saúde". O questionário ainda levantou informações socioeconômicas e demográficas sobre os entrevistados. Acrescenta-se que o projeto de pesquisa, o questionário e o termo de consentimento da pesquisa foram aprovados pelo Comitê de Ética em Pesquisa da Faculdade de Ciências Médicas (FCM) da Universidade Estadual de Campinas (UNICAMP).

Os atributos citados foram agrupados em categorias. Foram calculadas as freqüências simples e relativas dos atributos dos alimentos citados como prejudiciais à saúde. Realizou-se o teste de quiquadrado $\left(\chi^{2}\right)$ e o cálculo de resíduos padronizados ( $Z$ res.). O teste de qui-quadrado $\left(\chi^{2}\right)$ buscou identificar a existência de diferenças nas citações entre os grupos de atributos de alimentos considerados prejudiciais à saúde e entre as variáveis socioeconômicas e demográficas, bem como estimar, para cada um dos grupos de atributos de alimentos, as diferenças significativas entre os seus atributos e entre as variáveis socioeconômicas e demográficas. Nos testes de qui-quadrado $\left(\chi^{2}\right)$ foram adotados três níveis de significância, 10\%, 5\% e 1\%. Esses níveis de significância indicaram que é possível afirmar com uma confiabilidade de $90 \%$, $95 \%$ e $99 \%$, respectivamente, se realmente existe uma diferença significativa entre itens analisados.

Uma outra ferramenta estatística utilizada foi o cálculo dos resíduos padronizados ( $Z$ res.), que se revela muito útil para a elaboração de análise pormenorizada de cada uma das variáveis consideradas nas análises envolvendo o teste de quiquadrado. Através desse cálculo é possível verificar se um determinado atributo do alimento recebeu mais ( $Z$ res. positivo) ou menos ( $Z$ res. negativo) citações do que o esperado ${ }^{4}$. $\mathrm{Na}$ análise dos $Z$ res. foi adotado o nível de significância de $5 \%$, que corresponde ao $Z$ res. maior que 1,96 ou menor que -1,96. Destaca-se que o procedimento de categorização foi realizado de forma a agrupar o maior número de elementos em cada item; contudo, ao avaliar o número de citações de acordo com as características socioeconômicas e demográficas dos entrevistados, alguns itens apresentaram um número reduzido de citações. Para esses casos, não foi considerado no trabalho o resultado do teste $Z$ res.

\section{Resultados e discussão}

Perfil socioeconômico e demográfico dos entrevistados

Os dados relativos às características socioeconômicas e demográficas dos entrevistados foram reunidos na Tabela 1 . Integraram a pesquisa 75 homens $(47,5 \%)$ e 83 mulheres (52,5\%). De forma geral, com relação à idade, dois intervalos de idade são predominantes: menor ou igual a 25 anos (39,2\%) e com mais de 25 a 40 anos $(37,3 \%)$.

Quanto à escolaridade, cerca de $40 \%$ e de $50 \%$ dos entrevistados, cursaram, respectivamente, o ensino fundamental e o ensino médio, sendo que cerca de $50 \%$ dos homens e $48 \%$ das mulheres cursaram o ensino médio. Com relação à variável renda, a maioria dos entrevistados pertencia ao estrato de dois a dez salários mínimos (52,5\%), sendo que os homens apresentaram renda domiciliar média (6,7 salários mínimos) mais elevada, quando comparada à renda média das mulheres (5,7 salários mínimos). A renda domiciliar média dos entrevistados foi de 6,2 salários mínimos e o número médio de pessoas foi de 4,1 por domicílio e, portanto, a renda per capita média foi de 1,5 salários mínimos.

Opinião dos consumidores relativa aos atributos dos alimentos, considerados prejudiciais a saúde

Os entrevistados citaram cerca de 20 tipos diferentes de atributos dos alimentos considerados prejudiciais à saúde. Esses atributos foram distribuídos em quatro grupos distintos, a saber: nutrientes e componentes dos alimentos, biológicos, químicos e aspectos gerais (Tabela 2).

Examinando os dados da Tabela 2 pode-se observar que o grupo de atributos mais mencionado pelos indivíduos foi o de nutrientes e componentes dos alimentos (49,8\%), e esse grupo também reúne os atributos mais citados pelos indivíduos, como a gordura $(24,3 \%)$ e o colesterol $(11,3 \%)$.

\footnotetext{
${ }^{3}$ O cálculo do valor esperado de citações é realizado levando em consideração que nenhum dos grupos de entrevistados estaria mais ou menos propensos a se preocupar com um atributo do alimento, comparativamente a outro.
} 
Opinião de consumidores e riscos alimentares, Fonseca et al.

Tabela 1. Características socioeconômicas e demográficas dos consumidores. Município de Campinas, 2001

\begin{tabular}{|c|c|c|c|c|c|c|}
\hline \multirow{2}{*}{$\begin{array}{l}\text { Variáveis } \\
\text { Idade (anos) }\end{array}$} & \multicolumn{2}{|c|}{ Masculino } & \multicolumn{2}{|c|}{ Feminino } & \multicolumn{2}{|c|}{ Total } \\
\hline & $\mathbf{N}^{0}$ & $\%$ & $\mathbf{N}^{0}$ & $\%$ & $\mathbf{N}^{0}$ & $\%$ \\
\hline Menor ou igual a 25 & 19 & 25,3 & 43 & 51,8 & 62 & 39,2 \\
\hline Mais de 25 a 40 & 29 & 38,7 & 30 & 36,1 & 59 & 37,3 \\
\hline Mais de 40 a 55 & 18 & 24,0 & 6 & 7,2 & 24 & 15,2 \\
\hline Mais de 55 & 9 & 12,0 & 4 & 4,8 & 13 & 8,2 \\
\hline Total & 75 & 100,0 & 83 & 100,0 & 158 & 100,0 \\
\hline Média (anos) & 36,4 & & 28,2 & & 32,1 & \\
\hline \multicolumn{7}{|l|}{ Escolaridade } \\
\hline Sem Instrução & 1 & 1,3 & 1 & 1,2 & 2 & 1,3 \\
\hline Ensino fundamental & 28 & 37,3 & 36 & 43,3 & 64 & 40,5 \\
\hline Ensino médio & 38 & 50,7 & 40 & 48,3 & 78 & 49,4 \\
\hline Superior & 8 & 10,7 & 6 & 7,2 & 14 & 8,9 \\
\hline Total & 75 & 100,0 & 83 & 100,0 & 158 & 100,0 \\
\hline \multicolumn{7}{|l|}{ Renda domiciliar (SM)* } \\
\hline até 1 & 1 & 1,3 & 3 & 3,6 & 4 & 2,5 \\
\hline 1 a 2 & 8 & 10,7 & 18 & 21,7 & 26 & 16,5 \\
\hline 2 a 5 & 21 & 28,0 & 22 & 26,5 & 43 & 27,2 \\
\hline 5 a 10 & 24 & 32,0 & 16 & 19,3 & 40 & 25,3 \\
\hline 10 a 20 & 13 & 17,3 & 9 & 10,8 & 22 & 13,9 \\
\hline Mais de 20 & 1 & 1,3 & 3 & 3,6 & 4 & 2,5 \\
\hline Sem renda (desempregado) & 4 & 5,3 & 3 & 3,6 & 7 & 4,4 \\
\hline Não sabe & 3 & 4,0 & 9 & 10,8 & 12 & 7,6 \\
\hline Total & 75 & 100,0 & 83 & 100,0 & 158 & 100,0 \\
\hline Média de pessoas na família & 3,8 & & 4,3 & & 4,1 & \\
\hline Média (SM) & 6,7 & & 5,7 & & 6,2 & \\
\hline Renda per capita média domiciliar & 1,8 & & 1,3 & & 1,5 & \\
\hline
\end{tabular}

(*) SM = 1 Salário mínimo, que na época da entrevista correspondia a R\$ 180,00.

Deve-se notar que os resultados desta pesquisa revelaram a crença espontânea dos consumidores com relação aos riscos dos alimentos. Entretanto, não se pode afirmar o grau de diferença do nível de preocupação dos indivíduos sobre diferentes atributos dos alimentos, que são constantemente pesquisados pela medição de atitudes (CLAYTON e GRIFFITH, 2003). 
Opinião de consumidores e riscos alimentares, Fonseca et al.

Tabela 2. Grupos de atributos dos alimentos considerados prejudiciais à saúde pelos consumidores. Município de Campinas, 2001

\begin{tabular}{|c|c|c|c|c|}
\hline Grupos de Atributos & $\mathrm{N}^{\mathrm{o}}$ & \% (no grupo) & $\%$ & Posi \\
\hline \multicolumn{5}{|c|}{ Nutrientes e componentes dos alimentos: } \\
\hline Gordura & 58 & 48,7 & 24,3 & \\
\hline Colesterol & 27 & 22,7 & 11,3 & \\
\hline Sal (Cloreto de Sódio) & 10 & 8,4 & 4,2 & \\
\hline Cafeína & 7 & 5,9 & 2,9 & \\
\hline Açúcar & 11 & 9,2 & 4,6 & \\
\hline Outros: acidez, glúten, etc. & 6 & 5,0 & 2,5 & \\
\hline Sub-total & 119 & 100,0 & 49,8 & \\
\hline
\end{tabular}

\section{Biológicos:}

Bactérias de forma geral 3

$18,8 \quad 1,3 \quad 12$

Bactérias: Salmonella.

18,8

$1,3 \quad 12$

Bactérias: clostridium botulinum

25,0

1,7

11

Vaca louca, tênia, fungos, etc.

25,0

1,7

11

Outros

12,5

0,8

13

Sub-total

100,0

6,7

3

\section{Químicos:}

Agrotóxicos

Conservantes/Corantes

Generalizados como químicos

Hormônios

Aromatizantes e antioxidantes

Outros: antibióticos

Sub-total

\section{Atributos gerais:}

Generalizados como industrializados 2

e modo de fabricação

Validade (produtos vencidos)

Qualidade

Procedência

Higiene/sanidade

Outros (contaminação)

Sub-total

TOTAL

$\begin{array}{cccc}15 & 28,3 & 6,3 & 5 \\ 15 & 28,3 & 6,3 & 5 \\ 10 & 18,9 & 4,2 & 7 \\ 4 & 7,5 & 1,7 & 11 \\ 2 & 3,8 & 0,8 & 13 \\ 6 & 13,2 & 2,5 & 9 \\ \mathbf{5 2} & \mathbf{1 0 0 , 0} & \mathbf{2 1 , 8} & \mathbf{2}\end{array}$


O destaque dado pelos entrevistados na pesquisa com relação aos riscos associados à composição da dieta (gordura, colesterol, entre outros) também foi observado em outros estudos realizados em diversas regiões do Brasil (FAZIO, 1997; SPERS,1998).

Algumas pesquisas realizadas no Brasil revelaram maior interesse do consumidor pelo preço dos produtos alimentícios e concluíram que, de forma geral, ainda é pequeno o interesse de parte da população brasileira por aspectos relacionados ao teor nutricional, à qualidade e à segurança dos alimentos (O NOVO PERFIL DO CONSUMIDOR,1992; SALGUEIRO, 1995 e BUSO 2000).

No cenário internacional envolvendo países cuja renda é considerada elevada, os consumidores mostraram grande grau de inquietação com os componentes nutricionais dos alimentos. Isso aparece claramente com a pesquisa de Lappalainen et al. (1998), realizada entre 1995 e 1996, com o acompanhamento de 14.331 pessoas de quinze países da Europa. Também o estudo de Bartlan (1993), tendo por base a amostra de 20.000 americanos, conduzido pela Nilsen Market Reseach e pela Hanley Center, e a pesquisa do Food Marketing Institute citado por Spers \& Kassouk (1995) com consumidores americanos, confirmam a tendência descrita acima.

A pesquisa de Becker et al. (2000) mostra que os consumidores alemães não se preocupam muito com a gordura ou com o colesterol presentes na carne bovina. Oltersdorf (1993) já havia constatado esse comportamento dos alemães com a composição nutricional dos alimentos na sua pesquisa divulgada em 1993 e descrita no trabalho de revisão publicado por Wandel (1994).

Porém, em diversos países as pesquisas descrevem grande preocupação dos entrevistados com a gordura e o colesterol dos alimentos. Esses estudos internacionais realizados com os consumidores explicam os motivos que influenciam essa preocupação, que são entre outros: as mudanças e a divulgação das recomendações nutricionais, sugerindo a redução da ingestão de gorduras; as propagandas das indústrias alimentícias sobre produtos com reduzido teor de gordura e colesterol; e a veiculação pela mídia de resultados de estudos sobre a relação entre dieta e doenças (BARTLAN, 1993; RALPH et al., 1996; CRANE et al. 1999; HAMILTON et al., 2000).

Ainda com base nos dados da Tabela 2, é importante ressaltar que os entrevistados também mencionaram o grupo dos atributos químicos (21,8\%), destacando-se os agrotóxicos (6,3\%), os conservantes/corantes $(6,3 \%)$ e os produtos químicos em geral (4,2\%). No Brasil, ainda são reduzidos os estudos que avaliam a percepção dos consumidores com relação aos produtos químicos usados nos alimentos. Contudo, Spers (1998) realizou pesquisa com consumidores de produtos comercializados em feiras orgânicas e tradicionais e pôde constatar que a certificação orgânica não foi considerada de grande importância pelos entrevistados.

A preocupação de consumidores com os aspectos relacionados aos atributos químicos e tecnológicos dos alimentos é expressiva em muitos países. Segundo diversos autores, os contaminantes e os aditivos atraíram muito mais a atenção do que o esperado, sendo considerados pelos consumidores como um perigo e uma ameaça à vida humana. Esse fato é relatado em vários artigos que analisaram a "neofobia à tecnologia" (LEE,1989; OT'T et al. ,1991; SPARKS, 1994; MERTZ et al. 1998; TUORILA et al., 2001).

Ott et al. (1991) observaram que cerca de 55\% dos entrevistados revelaram apreensão com o uso de pesticidas nos alimentos, sendo que 30\% desses indivíduos disseram ser essa sua preocupação principal. Eles destacam que os consumidores em geral têm uma percepção diferente da dos especialistas da área de alimentos e nutrição. Segundo Lee (1989) os especialistas preocupam-se, em ordem decrescente de importância, com os seguintes perigos: microbiológicos, composição da dieta (tendo em vista à obesidade), contaminantes ambientais, toxinas naturais, substâncias químicas agrícolas (pesticidas) e aditivos; contudo, o público em geral revela maior preocupação com as substâncias químicas (LEE, 1989; SENAUER, 1991; MERTZ et al., 1998; TUORILA et al., 2001).

Entre os grupos de atributos dos alimentos identificados nesta pesquisa, os perigos biológicos foram os menos citados pelos entrevistados, quando se considera que este grupo recebeu apenas $6,7 \%$ das 
citações dos entrevistados (Tabela 2). Na verdade, Praxedes (2003) observou que era precário o conhecimento de uma comunidade de São Paulo em relação a microorganismos e sua transmissão pelos alimentos. Contudo, quando se analisa resultados de estudos internacionais observa-se uma expressiva preocupação dos entrevistados com os riscos biológicos. Por exemplo, na pesquisa de Ott et al. (1991) foi observado que cerca de $36 \%$ dos entrevistados estavam preocupados com as toxinfecções, sendo que esse item ocupou a segunda posição na ordenação elaborada pelos autores.

Também foi observado na presente pesquisa que os entrevistados revelaram interesse pelos atributos relacionados à segurança dos alimentos, como a qualidade
$(7,9 \%)$ e a higiene $(6,7 \%)$, que ocupam a terceira e quarta posições, respectivamente no rol dos atributos dos alimentos citados como prejudiciais à saúde.

\section{Variáveis socioeconômicas e demográficas e atributos considerados} prejudiciais à saúde pelos entrevistados na pesquisa.

Várias pesquisas, como as de Jussane \& Judson (1992), Moon (1998), Verbeke (2000) destacam a influência das características socioeconômicas e demográficas na percepção do risco e no comportamento de compra dos consumidores. Por isso, é importante discutir a relação dos atributos dos alimentos considerados prejudiciais à saúde pelos consumidores, associando-a às variáveis socioeconômicas e demográficas da amostra de

Tabela 3. Análise de resíduos padronizados (Z Res.) e Teste de qui-quadrado $\left(\chi^{2}\right)$ entre os grupos de atributos de alimentos e as variáveis socioeconômicas e demográficas. Município de Campinas, 2001

\begin{tabular}{|c|c|c|c|c|c|c|c|c|c|}
\hline \multirow{2}{*}{$\begin{array}{l}\text { Variáveis } \\
\text { Gênero }\end{array}$} & \multicolumn{2}{|c|}{$\begin{array}{c}\text { Nutrientes e componentes } \\
\text { dos Alimentos }\end{array}$} & \multicolumn{2}{|c|}{ Biológicos } & \multicolumn{2}{|c|}{ Químicos } & \multicolumn{2}{|c|}{ Outros } & \multirow[t]{2}{*}{$\chi^{2 * *}$} \\
\hline & Z Res*. & $\chi^{2 * *}$ & Z Res*. & $\chi^{2 * *}$ & Z Res*. & $\chi^{2 * *}$ & Z Res*. & $\chi^{2 * *}$ & \\
\hline Masculino & $-3,19$ & \multirow[b]{2}{*}{0,683} & $-1,14$ & \multirow[b]{2}{*}{0,089} & 2,91 & \multirow[b]{2}{*}{0,710} & 1,65 & \multirow[b]{2}{*}{0,374} & \multirow[b]{2}{*}{0,002} \\
\hline Feminino & 3,19 & & 1,14 & & $-2,91$ & & $-1,65$ & & \\
\hline \multicolumn{10}{|l|}{ Idade (anos) } \\
\hline Menor ou igual de 25 & 2.40 & \multirow{4}{*}{0,078} & $-0,29$ & \multirow{4}{*}{0,647} & $-2,73$ & \multirow{4}{*}{0,671} & 0,01 & \multirow{4}{*}{0,727} & \multirow{4}{*}{0,031} \\
\hline Mais de 25 a 40 & $-1,48$ & & 1,90 & & 0,89 & & $-0,26$ & & \\
\hline Mais de 40 a 55 & 1,79 & & 1,93 & & 2,23 & & 1,10 & & \\
\hline Mais de 55 & 1,02 & & $-0,20$ & & 0,01 & & $-1,13$ & & \\
\hline \multicolumn{10}{|l|}{ Escolaridade } \\
\hline Ensino fundamental & 0,91 & \multirow{3}{*}{0,082} & 0,86 & \multirow{3}{*}{0,422} & $-0,21$ & \multirow{3}{*}{0,360} & 0,79 & \multirow{3}{*}{0,398} & \multirow{3}{*}{0,920} \\
\hline Ensino médio & 0,73 & & $-0,54$ & & $-0,12$ & & $-0,43$ & & \\
\hline Ensino superior & 0,24 & & $-0,47$ & & $-0,53$ & & $-0,53$ & & \\
\hline \multicolumn{10}{|c|}{ Renda domiciliar (SM)* } \\
\hline até 2 & 1,49 & \multirow{4}{*}{0,515} & 0,39 & \multirow{4}{*}{0,171} & $-1,27$ & \multirow{3}{*}{0,286} & $-0,77$ & \multirow{4}{*}{0,646} & \multirow{4}{*}{0,088} \\
\hline 2 a 5 & $-1,78$ & & 1,73 & & $-0,06$ & & 1,15 & & \\
\hline 5 a 10 & $-1,22$ & & $-0,71$ & & 2,44 & & $-0,53$ & & \\
\hline Mais de 10 & 2,03 & & $-1,41$ & & $-1,64$ & & 0,05 & & \\
\hline
\end{tabular}

*Z Res. o nível de significância adotado foi de $5 \%$, que corresponde ao Z res. maior que 1,96 ou menor que $-1,96$;

** Teste de qui-quadrado $(\chi 2)$ significativo nos níveis de $10 \%, 5 \%$ ou de $1 \%$, respectivamente. 
consumidores.

De forma geral, a Tabela 3 mostra que existem diferenças de opinião dos entrevistados sobre os riscos alimentares, de acordo com o gênero, a idade e a renda domiciliar. Com relação ao gênero dos entrevistados, as diferenças observadas, identificadas mediante cálculo de resíduos padronizados, concentram-se principalmente no grupo dos nutrientes e componentes dos alimentos. É possível observar pela Tabela 4 que as mulheres $(61,8 \%)$ citaram bem mais que os homens (40,9\%), o grupo dos nutrientes e componentes dos alimentos. Esse interesse maior das mulheres também foi relatado nos trabalhos de Wandel (1994), Ralph (1996), Papp et al. (1997), Moon et al (1998).

Ainda, tendo por base a Tabela 4, cabe destacar que entre os homens foi captada maior preocupação em relação ao perigo químico (28,5\% de citações), quando comparada à das mulheres (12,7\% de citações). Neste caso, os homens destacaram especialmente os agrotóxicos, os conservantes e corantes e os produtos químicos em geral.

O cálculo de resíduos padronizados (Tabela 4) detectou que a qualidade dos alimentos foi mais mencionada por homens $(45,7 \%)$ do que por mulheres $(3,6 \%)$, fato que determina diferenças significativas. Ressalta-se também que a questão da higiene e sanidade dos alimentos é proporcionalmente mais mencionada pelos homens $(25,7 \%)$ do que pelas mulheres $(8,4 \%)$; entretanto para esse atributo de alimentos não foi detectada diferença significativa segundo os testes realizados.

Então foi possível verificar que no levantamento da opinião espontânea junto aos homens, os perigos químicos, a presença de bactérias e a qualidade dos alimentos são mais freqüentemente citados, enquanto que entre as mulheres as citações relacionam-se, predominantemente, com o grupo de nutrientes e componentes dos alimentos. Portanto, observa-se que ambos os gêneros mostram preocupação com aspectos específicos da segurança do alimento, como os perigos químicos e a composição nutricional dos alimentos. Contudo, a grande maioria dos estudos afirma que as mulheres revelam maior preocupação com a segurança do alimento ou com os riscos alimentares à saúde do que os indivíduos do gênero masculino (SCHAFER et al,1993; SJÖDEN, apud WANDEL, 1994; FREWER, 2001 e WARLAND et al., 2001).

A Tabela 5 apresenta os grupos de atributos, bem como os atributos dos alimentos que foram considerados prejudiciais à saúde pelos entrevistados, segundo os estágios de vida, analisados com adoção de técnica estatística (teste de qui-quadrado). Observa-se pela Tabela 3 que apenas o grupo de nutrientes e componentes dos alimentos apresentou diferença estatisticamente significativa. Adotando-se o cálculo de resíduos padronizados verifica-se que a gordura foi o atributo que mais influenciou essa diferença, pois, proporcionalmente, foi mais citada pelos indivíduos com idade inferior a 25 anos (60\%, Z res. positivo) e pelos indivíduos com mais de 55 anos $(63,6 \%)$ (Tabela 5).

Alguns estudos (SJÖDEN, apud WANDEL, 1994; JUSSAME \& JUDSON, 1992) registram que as pessoas mais velhas são as que revelam maior preocupação com a segurança dos alimentos. Os resultados da presente pesquisa indicam uma preocupação dos indivíduos com mais de 55 anos com relação a elementos como a gordura e o colesterol, que constituem potencialmente um perigo para pessoas que se encontram nesse estágio de vida. Vale mencionar que a gordura $(63,6 \%)$ e o colesterol $(27,3 \%)$ foram os atributos mais mencionados por esses indivíduos (Tabela 5).

A Tabela 5 mostra que existe variação na freqüência de citações dos indivíduos classificados em diferentes estágios de vida com relação aos atributos. O grupo perigos químicos foi mencionado mais pelos entrevistados com mais de 40 a 55 anos de idade (34,9\% de citações), enquanto que o grupo perigos biológicos foi mais mencionado por pessoas com mais de 25 a 40 anos idade. Cabe ressaltar que as pessoas nesse estágio de vida citaram mais a questão da higiene e sanidade dos alimentos.

Examinando os dados das Tabelas 3 e 6, observase que o grau de escolaridade não influenciou muito a opinião dos entrevistados. Contudo, do grupo de nutrientes e componentes dos alimentos, o atributo sal foi mais citado pelos indivíduos que possuem apenas o nível fundamental, conforme mostra a Tabela 6. Nota-se também que com relação ao grupo de perigos químicos, os indivíduos com maior escolaridade citaram de forma predominante os conservantes e corantes $(50 \%)$ como elementos perigosos à saúde, o que não se verificou com os 
Tabela 4. Atributos dos alimentos considerados prejudiciais à saúde, de acordo com o gênero dos entrevistados. Município de Campinas, 2001

\begin{tabular}{|c|c|c|c|c|}
\hline \multirow{3}{*}{$\begin{array}{l}\text { Grupos de Atributos } \\
\text { Nutrientes e componentes dos alimentos }\end{array}$} & \multicolumn{4}{|c|}{ Gênero } \\
\hline & \multicolumn{2}{|c|}{ Masculino } & \multicolumn{2}{|c|}{ Feminino } \\
\hline & $\mathrm{N}^{\circ}$ & $\%$ & $\mathrm{~N}^{\mathrm{o}}$ & $\%$ \\
\hline Gordura & 27 & 48,2 & 31 & 49,2 \\
\hline Colesterol & 15 & 26,8 & 12 & 19,0 \\
\hline Sal (Cloreto de Sódio) & 5 & 8,9 & 5 & 7,9 \\
\hline Cafeína & 3 & 5,4 & 4 & 6,3 \\
\hline Açúcar & 5 & 8,9 & 6 & 9,5 \\
\hline Outros: acidez, glúten, etc. & 1 & 1,8 & 5 & 7,9 \\
\hline Total (no grupo) & 56 & 100,0 & 63 & 100,0 \\
\hline Total & 56 & 40,9 & 63 & 61,8 \\
\hline \multicolumn{5}{|l|}{ Biológicos } \\
\hline Bactérias de forma geral & 3 & $42,9^{z+}$ & 0 & $0,0^{z-}$ \\
\hline Bactérias: salmonella. & 0 & 0,0 & 3 & 33,3 \\
\hline Bactérias: clostridium botulinum & 2 & 28,6 & 2 & 22,2 \\
\hline Outros: vaca louca, tênia, fungos, etc. & 2 & 28,6 & 4 & 44,4 \\
\hline Total (no grupo)* & 7 & 100 & 9 & 100 \\
\hline Total & 7 & 5,1 & 9 & 8,8 \\
\hline \multicolumn{5}{|l|}{ Químicos } \\
\hline Agrotóxicos & 11 & 28,2 & 4 & 30,8 \\
\hline Conservantes/Corantes & 10 & 25,6 & 5 & 38,5 \\
\hline Generalizados como químicos & 7 & 17,9 & 3 & 23,1 \\
\hline Hormônios & 4 & 10,3 & 0 & 0,0 \\
\hline Aromatizantes e antioxidantes & 2 & 5,1 & 0 & 0,0 \\
\hline Outros: antibióticos, etc. & 5 & 12,8 & 1 & 7,7 \\
\hline Total (no grupo) & 39 & 100,0 & 13 & 100,0 \\
\hline Total & 39 & 28,5 & 13 & 12,7 \\
\hline \multicolumn{5}{|l|}{ Atributos gerais } \\
\hline Generalizados como Industrializados/modo de fabricação & 1 & 2,9 & 1 & 5,9 \\
\hline Validade (produtos vencidos) & 3 & 8,6 & 3 & 3,6 \\
\hline Qualidade & 16 & $45,7^{z+}$ & 3 & $3,6^{z-}$ \\
\hline Procedência & 4 & 11,4 & 1 & 1,2 \\
\hline Higiene ou sanidade & 9 & 25,7 & 7 & 8,4 \\
\hline Outros (contaminação) & 2 & 5,7 & 2 & 2,4 \\
\hline Total (no grupo) & 35 & 100,0 & 17 & 25,2 \\
\hline Total & 35 & 25,5 & 17 & 16,7 \\
\hline
\end{tabular}

$*, * * \boldsymbol{o u} * * *=$ Teste de qui-quadrado $(\chi 2)$ significativo nos níveis de $10 \%, 5 \%$ ou de $1 \%$, respectivamente.

$\mathbf{z}^{+}=$Resíduo padronizado significativo e de valor positivo;

$\mathbf{z}^{-}=$Resíduo padronizado significativo e de valor negativo 
Opinião de consumidores e riscos alimentares, Fonseca et al.

Tabela 5. Atributos dos alimentos considerados prejudiciais à saúde pelos entrevistados, de acordo com o estágio de vida. Município de Campinas, 2001

\begin{tabular}{|c|c|c|c|c|c|c|c|c|}
\hline \multirow{3}{*}{$\begin{array}{l}\text { Atributos dos Alimentos } \\
\text { Nutrientes e componentes dos } \\
\text { Alimentos }\end{array}$} & \multicolumn{8}{|c|}{ Idade (anos) } \\
\hline & \multicolumn{2}{|c|}{ Menor ou igual a 25} & \multicolumn{2}{|c|}{ Mais 25 a 40} & \multicolumn{2}{|c|}{ Mais 40 a 55} & \multicolumn{2}{|c|}{ Mais de 55} \\
\hline & $\mathrm{N}^{\mathrm{o}}$ & $\%$ & $\mathrm{~N}^{\mathrm{o}}$ & $\%$ & $\mathrm{~N}^{\mathrm{o}}$ & $\%$ & $\mathrm{~N}^{\mathrm{o}}$ & $\%$ \\
\hline Gordura & 30 & $60,0^{2+}$ & 16 & 38,1 & 5 & 31,3 & 7 & 63,6 \\
\hline Colesterol & 12 & 24,0 & 10 & 23,8 & 2 & 12,5 & 3 & 27,3 \\
\hline Sal (Cloreto de Sódio) & 5 & 10,0 & 2 & 4,8 & 3 & 18,8 & 0 & 0,0 \\
\hline Cafeína & 2 & 4,0 & 3 & 7,1 & 2 & 12,5 & 0 & 0,0 \\
\hline Açúcar & 1 & $2,0^{z-}$ & 6 & 14,3 & 3 & 18,8 & 1 & 9,1 \\
\hline Outros: acidez, glúten, etc. & 0 & $0,0^{z-}$ & 5 & $11,9^{z+}$ & 1 & 6,3 & 0 & 0,0 \\
\hline Total* (no grupo) & 50 & 100,0 & 42 & 100,0 & 16 & 100,0 & 11 & 100,0 \\
\hline Total & 50 & 60,2 & 42 & 43,8 & 16 & 37,2 & 11 & 61,1 \\
\hline \multicolumn{9}{|l|}{ Biológicos } \\
\hline Bactérias de forma geral & 2 & 40,0 & 1 & 10,0 & 0 & 0,0 & 0 & 0,0 \\
\hline Bactérias: salmonella. & 1 & 20,0 & 2 & 20,0 & 0 & 0,0 & 0 & 0,0 \\
\hline Bactérias: clostridium botulinum & 1 & 20,0 & 2 & 20,0 & 0 & 0,0 & 1 & 100,0 \\
\hline Vaca louca, tênia, fungos, etc. & 1 & 20,0 & 3 & 30,0 & 0 & 0,0 & 0 & 0,0 \\
\hline Outros & 0 & 0,0 & 2 & 20,0 & 0 & 0,0 & 0 & 0,0 \\
\hline Total (no grupo) & 5 & 100,0 & 10 & 100,0 & 0 & 0,0 & 1 & 100,0 \\
\hline Total & 5 & 6,0 & 10 & 10,4 & 0 & 0,0 & 1 & 5,6 \\
\hline \multicolumn{9}{|l|}{ Químicos } \\
\hline Agrotóxicos & 3 & 30,0 & 7 & 29,2 & 4 & 26,7 & 1 & 25,0 \\
\hline Conservantes/Corantes & 4 & 40,0 & 6 & 25,0 & 4 & 26,7 & 1 & 25,0 \\
\hline Generalizados como químicos & 3 & 30,0 & 5 & 20,8 & 2 & 13,3 & 0 & 0,0 \\
\hline Hormônios & 0 & 0,0 & 2 & 8,3 & 2 & 13,3 & 0 & 0,0 \\
\hline Aromatizantes e antioxidantes & 0 & 0,0 & 1 & 4,2 & 0 & 0,0 & 1 & $25,0^{z+}$ \\
\hline Outros: antibióticos, etc. & 0 & 0,0 & 2 & 8,3 & 3 & 20,0 & 1 & 25,0 \\
\hline Total (no grupo) & 10 & 100,0 & 24 & 100,0 & 15 & 100,0 & 4 & 100,0 \\
\hline Total & 10 & 12,0 & 24 & 25,0 & 15 & 34,9 & 4 & 22,2 \\
\hline
\end{tabular}

\section{Atributos gerais}

Generalizados como Industrializados/

modo de fabricaço

Validade (produtos vencidos)

Qualidade

Procedência

Higiene ou sanidade

Outros (contaminação)

Total (no grupo)

Total

\begin{tabular}{cccccccc}
0 & 0,0 & 1 & 5,0 & 1 & 8,3 & 0 & 0,0 \\
3 & 16,7 & 2 & 10,0 & 1 & 8,3 & 0 & 0,0 \\
6 & 33,3 & 6 & 30,0 & 5 & 41,7 & 2 & 100,0 \\
2 & 11,1 & 2 & 10,0 & 1 & 8,3 & 0 & 0,0 \\
6 & 33,3 & 8 & 40,0 & 2 & 16,7 & 0 & 0,0 \\
1 & 5,6 & 1 & 5,0 & 2 & 16,7 & 0 & 0,0 \\
$\mathbf{1 8}$ & $\mathbf{1 0 0 , 0}$ & $\mathbf{2 0}$ & $\mathbf{1 0 0 , 0}$ & $\mathbf{1 2}$ & $\mathbf{1 0 0 , 0}$ & $\mathbf{2}$ & $\mathbf{1 0 0 , 0}$ \\
$\mathbf{1 8}$ & $\mathbf{2 1 , 7}$ & $\mathbf{2 0}$ & $\mathbf{2 0 , 8}$ & $\mathbf{1 2}$ & $\mathbf{2 7 , 9}$ & $\mathbf{2}$ & $\mathbf{1 1 , 0}$ \\
\hline
\end{tabular}

$*$, $*$ ou $* * *=$ Teste de qui-quadrado $(\chi 2)$ significativo nos níveis de $10 \%, 5 \%$ ou de $1 \%$, respectivamente

$\mathbf{z}^{+}=$Resíduo padronizado significativo e de valor positivo; $\quad \mathbf{z}^{-}=$Resíduo padronizado significativo e de valor negativo 
Tabela 6. Atributos dos alimentos considerados prejudiciais à saúde pelos entrevistados, de acordo o grau de escolaridade. Município de Campinas, 2001

\begin{tabular}{|c|c|c|c|c|c|c|}
\hline \multirow{2}{*}{$\begin{array}{l}\text { Atributos dos Alimentos } \\
\text { Nutrientes e componentes dos } \\
\text { Alimentos }\end{array}$} & \multicolumn{2}{|c|}{ Fundamental } & \multicolumn{2}{|c|}{ Médio } & \multicolumn{2}{|c|}{ Superior } \\
\hline & $\mathrm{N}^{\mathrm{o}}$ & $\%$ & $\mathrm{~N}^{\mathrm{o}}$ & $\%$ & $\mathrm{~N}^{\mathrm{o}}$ & $\%$ \\
\hline Gordura & 17 & 45,9 & 35 & 50,0 & 6 & 50,0 \\
\hline Colesterol & 7 & 18,9 & 18 & 25,7 & 2 & 16,7 \\
\hline Sal (Cloreto de Sódio) & 6 & $16,2^{z+}$ & 4 & 5,7 & 0 & 0,0 \\
\hline Cafeína & 2 & 5,4 & 5 & 7,1 & 0 & 0,0 \\
\hline Açúcar & 4 & 10,8 & 6 & 8,6 & 1 & 8,3 \\
\hline Outros: acidez, glúten, etc. & 1 & 2,7 & 2 & 2,9 & 3 & $25,0^{z+}$ \\
\hline Total* (no grupo) & 37 & 100,0 & 70 & 100,0 & 12 & 100,0 \\
\hline Total & 37 & 45,7 & 70 & 51,9 & 12 & 52,2 \\
\hline \multicolumn{7}{|l|}{ Biológicos } \\
\hline Bactérias de forma geral & 1 & 14,3 & 2 & 25,0 & 0 & 0,0 \\
\hline Bactérias: salmonella. & 1 & 14,3 & 1 & 12,5 & 1 & $100,0^{z+}$ \\
\hline Bactérias: clostridium botulinum & 1 & 14,3 & 3 & 37,5 & 0 & 0,0 \\
\hline Vaca louca, tênia, fungos, etc. & 2 & 28,6 & 2 & 25,0 & 0 & 0,0 \\
\hline Outros & 2 & 28,6 & 0 & 0,0 & 0 & 0,0 \\
\hline Total (no grupo) & 7 & 100,0 & 8 & 100,0 & 1 & 100,0 \\
\hline Total & 7 & 8,6 & 8 & 5,9 & 1 & 4,3 \\
\hline \multicolumn{7}{|l|}{ Químicos } \\
\hline Agrotóxicos & 4 & 23,5 & 10 & 34,5 & 1 & 16,7 \\
\hline Conservantes/Corantes & 5 & 29,4 & 7 & 24,1 & 3 & 50,0 \\
\hline Generalizados como químicos & 5 & 29,4 & 5 & 17,2 & 0 & 0,0 \\
\hline Hormônios & 0 & 0,0 & 3 & 10,3 & 1 & 16,7 \\
\hline Aromatizantes e antioxidantes & 1 & 5,9 & 0 & 0,0 & 1 & 16,7 \\
\hline Outros: antibióticos, etc. & 2 & 11,8 & 4 & 13,8 & 0 & 0,0 \\
\hline Total (no grupo) & 17 & 100,0 & 29 & 100,0 & 6 & 100,0 \\
\hline Total & 17 & 21,0 & 29 & 21,5 & 6 & 26,1 \\
\hline
\end{tabular}

\section{Atributos gerais}

Generalizados como Industrializados/

Validade (produtos vencidos)

Qualidade

Procedência

Higiene ou sanidade

Outros (contaminação)

Total (no grupo)

\begin{tabular}{cccccc}
0 & 0,0 & 2 & 7,1 & 0 & 0,0 \\
2 & 10,0 & 3 & 10,7 & 1 & 25,0 \\
10 & 50,0 & 7 & 25,0 & 2 & 50,0 \\
1 & 5,0 & 4 & 14,3 & 0 & 0,0 \\
5 & 25,0 & 11 & 39,3 & 0 & 0,0 \\
2 & 10,0 & 1 & 3,6 & 1 & 25,0 \\
20 & $\mathbf{1 0 0 , 0}$ & $\mathbf{2 8}$ & $\mathbf{1 0 0 , 0}$ & $\mathbf{4}$ & $\mathbf{1 0 0 , 0}$ \\
$\mathbf{2 0}$ & $\mathbf{2 4 , 7}$ & $\mathbf{2 8}$ & $\mathbf{2 0 , 7}$ & $\mathbf{4}$ & $\mathbf{1 7 , 4}$ \\
\hline
\end{tabular}

$*$, $*$ ou $* * *=$ Teste de qui-quadrado $(\chi 2)$ significativo nos níveis de $10 \%, 5 \%$ ou de $1 \%$, respectivamente.

$\mathbf{z}^{+}=$Resíduo padronizado significativo e de valor positivo;

$\mathbf{z}^{-}=$Resíduo padronizado significativo e de valor negativo. 
Opinião de consumidores e riscos alimentares, Fonseca et al.

Tabela 7. Atributos dos alimentos considerados prejudiciais à saúde pelos entrevistados, de acordo o nível de renda domiciliar. Município de Campinas, 2001

\begin{tabular}{|c|c|c|c|c|c|c|c|c|}
\hline \multirow{3}{*}{$\begin{array}{l}\text { Atributos dos Alimentos } \\
\text { Nutrientes e componentes dos } \\
\text { Alimentos }\end{array}$} & \multicolumn{8}{|c|}{ Renda em salários mínimos (SM) } \\
\hline & \multicolumn{2}{|c|}{ Até 2 SM } & \multicolumn{2}{|c|}{2 a $5 \mathrm{SM}$} & \multicolumn{2}{|c|}{5 a $10 \mathrm{SM}$} & \multicolumn{2}{|c|}{ Mais de $10 \mathrm{SM}$} \\
\hline & $\mathrm{N}^{\mathrm{o}}$ & $\%$ & $\mathrm{~N}^{\mathrm{o}}$ & $\%$ & $\mathrm{~N}^{\mathrm{o}}$ & $\%$ & $\mathrm{~N}^{\mathrm{o}}$ & $\%$ \\
\hline Gordura & 10 & 45,5 & 15 & 62,5 & 14 & 41,2 & 15 & 51,7 \\
\hline Colesterol & 3 & 13,6 & 5 & 20,8 & 8 & 23,5 & 7 & 24,1 \\
\hline Sal (Cloreto de Sódio) & 3 & 13,6 & 1 & 4,2 & 4 & 11,8 & 2 & 6,9 \\
\hline Cafeína & 2 & 9,1 & 2 & 8,3 & 2 & 5,9 & 0 & 0,0 \\
\hline Açúcar & 1 & 4,5 & 1 & 4,2 & 5 & 14,7 & 4 & 13,8 \\
\hline Outros: acidez, glúten, etc. & 3 & $13,6^{z+}$ & 0 & 0,0 & 1 & 2,9 & 1 & 3,4 \\
\hline Total* (no grupo) & 22 & 100,0 & 24 & 100,0 & 34 & 100,0 & 29 & 100,0 \\
\hline Total & 22 & 61.1 & 24 & 40,0 & 34 & 44,2 & 29 & 63,0 \\
\hline \multicolumn{9}{|l|}{ Biológicos } \\
\hline Bactérias de forma geral & 0 & 0,0 & 2 & 28,6 & 1 & 25,0 & 0 & 0,0 \\
\hline Bactérias: salmonella. & 1 & 33,3 & 1 & 14,3 & 0 & 0,0 & 0 & 0,0 \\
\hline Bactérias: clostridium botulinum & 0 & 0,0 & 1 & 14,3 & 2 & 50,0 & 1 & 100,0 \\
\hline Vaca louca, tênia, fungos, etc. & 0 & 0,0 & 3 & 42,9 & 1 & 25,0 & 0 & 0,0 \\
\hline Outros & 2 & $66,7^{z^{+}}$ & 0 & 0,0 & 0 & 0,0 & 0 & 0,0 \\
\hline Total (no grupo) & 3 & 100,0 & 7 & 100,0 & 4 & 100,0 & 1 & 100,0 \\
\hline Total & 3 & 8,3 & 7 & 11,7 & 4 & 5,2 & 1 & 2,2 \\
\hline \multicolumn{9}{|l|}{ Químicos } \\
\hline Agrotóxicos & 1 & 20,0 & 4 & 30,8 & 9 & 37,5 & 1 & 16,7 \\
\hline Conservantes/Corantes & 1 & 20,0 & 2 & 15,4 & 7 & 29,2 & 2 & 33,3 \\
\hline Generalizados como químicos & 3 & $60,0^{z+}$ & 3 & 23,1 & 3 & 12,5 & 1 & 16,7 \\
\hline Hormônios & 0 & 0,0 & 0 & 0,0 & 2 & 8,3 & 2 & $33,3^{z+}$ \\
\hline Aromatizantes e antioxidantes & 0 & 0,0 & 1 & 7,7 & 0 & 0,0 & 0 & 0,0 \\
\hline Outros: antibióticos, etc. & 0 & 0,0 & 3 & 23,1 & 3 & 12,5 & 0 & 0,0 \\
\hline Total (no grupo) & 5 & 100,0 & 13 & 100,0 & 24 & 100,0 & 6 & 100,0 \\
\hline Total & 5 & 13,9 & 13 & 21,7 & 24 & 31,2 & 6 & 13,0 \\
\hline \multicolumn{9}{|l|}{ Atributos gerais } \\
\hline $\begin{array}{l}\text { Generalizados como Industrializados/ } \\
\text { modo de fabricação }\end{array}$ & 1 & 16,7 & 0 & 0,0 & 1 & 6,7 & 0 & 0,0 \\
\hline Validade (produtos vencidos) & 0 & 0,0 & 2 & 12,5 & 3 & 20,0 & 1 & 10,0 \\
\hline Qualidade & 3 & 50,0 & 7 & 43,8 & 5 & 33,3 & 2 & 20,0 \\
\hline Procedência & 0 & 0,0 & 1 & 6,3 & 2 & 13,3 & 2 & 20,0 \\
\hline Higiene ou sanidade & 2 & 33,3 & 5 & 31,3 & 4 & 26,7 & 3 & 30,0 \\
\hline Outros (contaminação) & 0 & 0,0 & 1 & 6,3 & 0 & 0,0 & 2 & 20,0 \\
\hline Total (no grupo) & 6 & 100,0 & 16 & 100,0 & 15 & 100,0 & 10 & 100,0 \\
\hline Total & 6 & 16,7 & 16 & 26,7 & 15 & 19,5 & 10 & 21,7 \\
\hline
\end{tabular}

$*$ * $*$ ou $* * *$ Teste de qui-quadrado $(\chi 2)$ significativo nos níveis de $10 \%, 5 \%$ ou de $1 \%$, respectivamente

$\mathbf{z}^{+}=$Resíduo padronizado significativo e de valor positivo; $\mathbf{z}^{-}=$Resíduo padronizado significativo e de valor negativo 
entrevistados de ensino médio $(24,1 \%)$ (Tabela 6).

A Tabela 3 mostra como a renda domiciliar exerce influência na opinião dos entrevistados, no tocante aos grupos de atributos considerados prejudiciais à saúde. Neste caso, pode-se verificar que essa diferença significativa deve-se aos entrevistados com rendimentos superiores a 10 salários mínimos, que citaram prioritariamente o grupo de nutrientes e componentes dos alimentos e aos entrevistados com renda domiciliar entre cinco e dez salários mínimos que mencionaram predominantemente o grupo de atributos químicos. Ainda é possível verificar pela Tabela 7 que os perigos químicos foram mais citados pela faixa de renda de cinco a 10 salários mínimos $(31,2 \%)$ do que pela faixa de renda menor ou igual a dois salários mínimos $(13,9 \%)$.

$\mathrm{Na}$ literatura consultada obteve-se informações variadas da influência da escolaridade e dos rendimentos na percepção dos consumidores sobre a segurança dos alimentos. Jussame \& Judson (1992) mostraram que quanto maior o nível de renda e educação, maior é a preocupação dos consumidores com a segurança do alimento. Porém, estudo feito por Warland et al. (2001) ${ }^{1}$ revelou que os entrevistados com baixa educação e renda eram mais preocupados com a segurança dos alimentos. Fullmer et al. (1991) e Moon et al. (1998) perceberam que determinados grupos de indivíduos destacaram-se com relação à preocupação com algum grupo de atributos dos alimentos. Por exemplo, Moon et al. (1998) constataram que as pessoas com maiores rendimentos familiares tendem a revelar maior preocupação com os aspectos nutricionais dos alimentos. Os autores ainda mencionaram que as pessoas com maior escolaridade têm maior nível de conhecimento relativo às dietas e doenças crônico-degenerativas.

\section{Considerações Finais}

Os resultados deste estudo podem subsidiar a elaboração de políticas públicas de segurança do alimento, assim como as ações da indústria de alimentos. Neste estudo foi possível identificar que os consumidores citaram diferentes riscos dos alimentos com freqüência diferenciada. Observou-se maior freqüência de citações relativas aos componentes nutricionais da dieta, como por exemplo a gordura e o colesterol. Esses resultados refletem uma tendência mundial observada no comportamento dos consumidores, que se mostram mais atentos aos aspectos nutricionais da alimentação, visando à qualidade de vida.

Os entrevistados relataram também atributos gerais dos alimentos como procedência, qualidade, sanidade e perigos químicos, entre outros. Contudo, observa-se que não houve prevalência expressiva de citações relativas aos perigos microbiológicos, como por exemplo, a presença de bactérias patogênicas nos produtos alimentícios. Foi observada diferença estatisticamente significativa somente quando se comparou as freqüências de citações de atributos de alimentos considerados prejudiciais à saúde, levandose em consideração o gênero, a idade e os rendimentos domiciliares dos entrevistados.

Ressalta-se a importância de programas informativos e educacionais que podem ser desenvolvidos por órgãos governamentais, instituições, como é o caso das Universidades e Institutos de Pesquisas e pela própria indústria alimentícia, com o objetivo de diminuir o nível de insegurança das pessoas com relação às substâncias químicas utilizadas no processamento dos alimentos, às inovações tecnológicas do setor alimentício e a certos componentes dos alimentos. Acredita-se que essa preocupação é mais conseqüência de uma falta de compreensão sobre esses aspectos do que do real risco que eles representam.

A educação para o consumo também pode ser importante no controle da incidência das enfermidades transmitidas por alimentos, especialmente por meio de fatores biológicos, que vêm revelando crescimento em vários países, inclusive no Brasil. Se o consumidor for devidamente informado sobre os riscos da contaminação biológica e da incidência dessas enfermidades, ele poderá ampliar o seu nível de exigência quanto à segurança biológica dos produtos alimentícios e ainda adotar práticas de preparo e consumo de alimentos mais seguras. No entanto, é importante frisar que é responsabilidade do setor privado oferecer um alimento seguro, assim como é atribuição do governo o controle e a garantia de

\footnotetext{
${ }^{1}$ Warland (2001) descreve que os estudos de Becker (2000) e Horban (1996 e 1998) obtiveram resultados similares.
} 
segurança dos produtos disponíveis para o consumo.

\section{Referências Bibliográficas}

ASSOCIAÇÃO BRASILEIRA DE NORMAS TÉCNICA - ABNT. NBR 14900: sistema de gestão da análise de perigos e pontos críticos de controle Segurança de alimentos.Cidade, 2002. 9 p.

BARTLAN, M.J. Healthy and light foods: understanding the consumer. British Food Journal, Londres, v.95, n.3, p.3-11, 1993.

BECKER, T.; BENNER, E.; GLITSCH, K. Consumer perception of fresh meat quality in Germany. British Food Journal, Londres, v.102, n.3, p.246-266, 2000.

BRASIL, Conselho Nacional de Saúde. Resolução 196 de 10 de outubro de 1996.

BUSO, G. Análise do perfil do consumidor de carne bovina na cidade de São Paulo. São Carlos (SP). 2000. 174p. Dissertação (Mestrado em Administração) Departamento de Engenharia de Produção, Universidade Federal de São Carlos - UFSCar, São Carlos, 2000b.

CASWELL, J.A. Valuing food safety and nutrition. Boulder: Westview Pres, 1981, 457 p.

CHINNICI, G.; DÁMICO, M.; PECORINO, B. A multivariate statistical analysis on the consumers of organic products. British Food Journal, Londres, v. 104, n.3/4/5, p.187-199, 2002.

CLAYTON, D.A.; GRIFFITH, C.J.; PRICE, P. An investigation of the factors underlying consumers' implementation of specific food safety practices. London. British Food Journal, v.105, n.7, p.434453, 2003

COSTA, M.C.; DELIZA, R.; ROSENTHAL, A.; HEDDERLEY, D.; FREWER, L. Non conventional technologies and impact on consumer behavior. Food Science e Technology, v.11, s/d, p.188-193, 2000.

CRANE, N.T.; HUBBARD, V.S; LEWIS, C.J. American diets and year 2000 goals. In: FRAZAO, E. ed. America's Eating Habits: Changes and Consequences. Washington, DC: Economic Research Service, United States Dept of Agriculture; 1999. Agriculture
Information Bulletin No. 750:111-133

FAZIO, G.; FURQUIM, M.F.; KASSOUF, A.L. Preocupações dos consumidores com a qualidade de alimentos. Preços agrícolas, Campinas (SP), p. 912, 1997.

FREWER, L. Developing a food safety strategy. Diponível: http://www.who.org. 21 fev. 2001.

FULLMER, S.; GEIGER, C.J.; PARENT, C.R.M. Consumers' knowledge, understanding, and attitudes toward health claims on food labels, Journal of the American Dietetic Association, New Yourk, v.91, n. 2, 1991.

FUNDAÇÃO DO SISTEMA ESTADUAL DE ANÁLISES DE DADOS - SEADE, disponível: http://www.seade.gov.br/produtos/perfil/, acesso: 14/03/2005.

HAMILTON, J.; KNOX, B.; HILL, D.; PARR, H. Reduced fat products: consumer perception and preference. British Food Journal. Londres, v. 102, n. 7, p.494-506, 2000

INSTITUTO BRASILEIRO DE OPINIÃO PUBLICA E ESTATÍSTICA (IBOPE). Pesquisa de opinião pública sobre os transgênicos, São Paulo, 2002.

INSTITUTO BRASILEIRO DE OPINIÃO PUBLICA E ESTATÍSTICA (IBOPE). Pesquisa de opinião pública sobre os transgênicos, São Paulo, 2001.

JUSSAUME Jr., R.; JUDSON, D.H. Public perceptions about food safety in the United States and Japan. Rural Sociology, Montana, v.57, n.2, p.235-249, 1992.

LAPPALAINEN, R.; KEARNEY, J.; GIBNEY, M. A PAN EU survey of consumer attitudes to food, nutrition and health: an overview. Food Quality and Preference. Amsterdam,. v.9, n.6, p.467-478, 1998.

LEE, K. Food neophobia: major causes and treatments. Food Technology, Chicago, v.43, n.12, december, p.62-73, 1989

MERTZ, C.K.; SLOVIC, P.; PURCHASE, I.F.H Judgments of chemical risks: comparisons among 
senior managers, toxicologist, and the public. Risk Analysis, Londres, v.18, n.4, p.391-404, 1998

MOON, W.; FLORKOWSKI, W.J.; RESURRECCION, A. V. A.; BEUCHAT, L. R; CHINNAN, M.S.; PARASKOVA, P.; JORDANOV, J. Consumer concerns about nutritional attributes in a transition economy. Food Policy, Amsterdam, v.23, n. 5, p.357-369, 1998.

NELSON. R. Risk management behaviour by the Northern Ireland food consumer. International Journal of Consumer Studies, v. 28, n.2, p.186-193, 2004

O'DONOVAN, P.; McCARTHY, M. Irish consumer preference for organic meat. British Food Journal, Londres, v.104, n. 3/4/5, p. 353-370, 2002.

OTT, S.; HUANG, C.H; MISRA, S.K. Consumers' perceptions of risks from pesticide residues and demand for certification of residue-free produce. In: CASWELL, J.A.. Economic of food safety. New York: Elsevier Science Pub., 1991. Cap. 7 p.175-188.

O NOVO perfil do consumidor. Revista da Associação Brasileira de Supermercados. São Paulo, v.18, n.11, p.90-113, 1992.

PAPP, J.; LAKNER, Z.; KOMÁROMI, N.; LEHOTA, J. Food consumer types and behavior in Hugary: a suvery of food consumers' attitudes and practical behavior. ACTA Alimentaria, v. 26, n.3, p.199-217, 1997.

PRAXEDES, P.C.G.. Aspecto da qualidade higiênico-sanitária de alimentos consumidos e comercializados na comunidade São Remo, São Paulo, capital. 2003. 120p. Dissertação (Mestrado) Programa de Pós-graduação em Epidemilogia Experimental Aplicada à Zoonoses, Faculdade de Medicina e Veterinária e Zootecnia, Universidade de São Paulo, Piracicaba, 2003.

RALPH, L.; SEAMAN, C.E.A.; WOODS, M. Male attitudes towards healthy eating. British Food Journal. Londres, v.98, n.1, p.4-6, 1996.

REVISTA SUPER INTERESSANTE. Como o brasileiro se alimenta, ano 5, n. 6,7 e 8, 1991.

RIMAL,A.; FLETCHER, S.M.; McIWATTERS, K.H.; MISRA, S.K.; DEODHA, S. Perception of food safety and changes in food consumption habits: a consumer analysis. International Journal of Consumer Studies, v.25, n.1, po.43-52, 2001.

SABA, A.; ROSATI, S.; VASSALLO, M. Biotechnology in agriculture: perceived risks, benefits and attitudes in Italy. British Food Journal, Londres, v.102, n. 2, p. 114-121, 2000.

SALGUEIRO, S. Consumidor dá nota para os serviços de supermercados, SuperHiper, São Paulo, ano 21, n. 234, 1995

SCHAFER, R.; SCHAFER, E.; BULTENA, G.; HOIBERG, E.O. Food safety: applications of the health belief model. Journal of Nutrition Education. Canadá,. V. 25, N.1, P.17-23, 1993.

SENAUER, B. Consumer food safety concerns. Cereal Foods World, v.37, n.40, p.298-303, 1991.

SHINE, A.; O'REILLY, S.; O'SULLIVAN, K. Consumer attitudes to nutrition labeling, British Food Journal, Londres, v. 99, n. 8, p.283-289, 1997.

SPARKS, P.; SHEPHERD, R. Public perception of food-relatd hazards: individual and social dimensions. Food Quality and Preference. Amsterdam, v.5, n.3, p.185-194, 1994.

SPERS, E.E. Preferência do consumidor por atributos de segurança: aplicação de Conjoint Analysis. 1998. 108p. Dissertação (Mestrado) Programa de Pós-graduação em ciências da Economia Aplicada, Escola Superior de Agricultura "Luiz de Queiroz”, Universidade de São Paulo, Piracicaba, 1998.

SPERS, E.E.; Mecanismos de regulação da qualidade e segurança em alimentos. 2003. 136p. Tese (Doutorado) - Programa de Pós-graduação em Economia, Administração e Contabilidade, Faculdade Economia, Administração e Contabilidade Universidade de São Paulo, São Paulo, 2003.

SPERS, E. E.; KASSOUK, A.L. A abertura do mercado e a preocupação com a segurança alimentar sob o enfoque qualitativo, Brasília (DF), Anais do XXXIII congresso brasileiro de economia e sociologia rural, Curitiba (PR) 31 de julho a 3 de agosto de 1995.

TUORILA， H.; LÄHTEENMAÁKI， L.; POHJALAINEN, L.; LOTTI, L. Food neophobia among the finns and related responses to familiar and 
unfamiliar foods. Food Quality Preference, Amsterdam, v.12, n. , p.29-37, 2001.

VERBEKE, W. Influences on the consumer decisionmaking process towards fresh meat. British Food Journal, Londres, v.102, n.7, p.522-538, 2000

YEUNG, R.M.W.; MORRIS, J. Food safety risk: consumer perception and purchase behaviour. British Food Journal. Londres, v.103, n.3, p.170-186, 2001.
WANDEL, M. Understanding consumer concern about food-related health risks. British Food Journal. Londres, v.96, n.7, p.35-40, 1994.

WARLAND, R.H.; HERRMANN, R.O.; STERNGOLD, A. Assessing the bases of food safety concerns. In: Interdisciplinary Food Safety Research, Boca Raton: CRC Press, 2001, chapter 9, p. 183-203. 